\title{
The Case for Early Antibiotic Commencement and Source Control in Paediatric Subdural Empyema: A Single-Centre Retrospective Case Series
}

\author{
Fahid Tariq Rasul ${ }^{a}$ Aswin Chari ${ }^{a}$ b $\quad$ Mohammed Omar Iqbal ${ }^{a}$ Geeth Silva $^{a}$ \\ James Hatcher ${ }^{c}$ John Hartley ${ }^{c}$ Muhammad Zubair Tahir ${ }^{a, b}$ \\ a Department of Neurosurgery, Great Ormond Street Hospital, London, UK; ${ }^{b}$ Developmental Neurosciences, Great \\ Ormond Street Institute of Child Health, University College London, London, UK; 'Department of Microbiology, \\ Great Ormond Street Hospital, London, UK
}

\section{Keywords}

Subdural · Empyema · Surgery · Paediatric

\begin{abstract}
Background: Subdural empyema is a neurosurgical emergency requiring prompt diagnosis and treatment. There is a debate between the benefits and risks of starting early antibiotics prior to surgical drainage as this is purported to reduce the rate of microbiological diagnosis. Here, we describe our experience of treating this potentially life-threatening condition, advocating for the early commencement of antibiotics and importance of source control in its treatment. Methods: Retrospective review of a prospectively collected electronic departmental database included all patients who were admitted to our unit with a diagnosis of subdural empyema over an 11-year period (2008-2018). Basic demographic data were collected. Further data pertaining to mode of presentation, surgical approach, causative organism, post-operative antibiotic regime, anti-seizure medications, length of hospital stay, further surgery, and neurological outcomes were extracted. Results: Thirty-six children underwent 44 operations for subdural empyema at our institution during the study period. Median age was 11.0 (range $0.2-15.8) ; 47.2 \%$ (17/36) were female. Over time, there was decreasing use of burr holes and increasing use of craniec-
\end{abstract}

tomy as the index surgery. Using a combination of extended culture and polymerase chain reaction, a microbiological diagnosis was achieved in all 36 cases; the commonest causative microorganism was of the Streptococcus anginosus group of bacteria. Seven patients underwent repeat surgery, and 4 patients underwent a concurrent ENT procedure. No risk factors were significant in predicting the likelihood of reoperation (location of subdural empyema, age, index surgery type, inflammatory markers, concurrent ENT procedure, and microorganism) although it was notable that none of the patients undergoing a concurrent ENT procedure underwent repeat surgery $(p=0.29)$. Median length of stay was 12 days (range 3-74), and there were no inpatient or procedure-related mortalities. Clinical outcomes were good with 94.4\% (34/36) categorized as modified Rankin Scale 0-3 at discharge and there were 2 cranioplasty-related complications. Conclusions: We observed an evolution of practice from limited surgical approaches towards more extensive index surgery over the study period. Given that a microorganism was isolated in all cases using a comprehensive approach, initiation of antibiotic therapy should not be delayed on presentation. Concurrent ENT surgery may be an important factor in providing aggressive source control thereby reducing the need for repeat surgery.

(c) 2021 The Author(s)

Published by S. Karger AG, Basel karger@karger.com www.karger.com/pne

Karger $\stackrel{\text { ' }}{5}$

BOPEN ACCESS
(C) 2021 The Author(s)

Published by S. Karger AG, Basel

This article is licensed under the Creative Commons Attribution 4.0 International License (CC BY) (http://www.karger.com/Services/ OpenAccessLicense). Usage, derivative works and distribution are permitted provided that proper credit is given to the author and the original publisher.
Correspondence to:

Aswin Chari, aswinchari@gmail.com

Muhammad Zubair Tahir, zubair.tahir@gosh.nhs.uk 


\section{Introduction}

The neurosurgical community has long accepted the serious nature of subdural empyema, a purulent collection between the dura mater and arachnoid $[1,2]$. It is a neurosurgical emergency requiring prompt diagnosis and early treatment. It is often caused by either bacterial meningitis in infants or from sinusitis or otitis media in older children $[2,3]$. Although rare, it is associated with serious sequelae including venous sinus thrombosis/ stroke, cerebral oedema, and seizures. Mortality is approximately $4 \%$, and up to $35 \%$ of patients have a residual neurologic deficit [2].

Treatment generally involves emergency evacuation of the infected subdural collection followed by a longterm course of antibiotics. The aim of surgical treatment is to evacuate the pus but also, importantly, to obtain microbiological samples to guide definitive antibiotic treatment [3]. There is uncertainty as to when to start the antibiotics; whilst some advocate for early initiation of antibiotic, there is the argument that this could reduce the yield of the microbiological diagnosis, affecting the targeting of antibiotic treatment. The aim of this study was to evaluate the contemporary outcomes of paediatric subdural empyema, with specific focus on the surgical approaches, microbiological diagnoses, and risk factors for needing repeat surgical intervention.

\section{Methods}

We used the Strengthening the Reporting of Observational Studies in Epidemiology (STROBE) for this section of the manuscript.

\section{Study Design}

This was a retrospective cohort study including all children aged 17 years or less undergoing surgical intervention for subdural empyema.

\section{Setting and Participants}

The study was conducted at Great Ormond Street Hospital (GOSH) for Children, London, UK. This is the tertiary referral centre for paediatric patients with neurosurgical pathology in North London. All cases are entered onto a prospectively maintained database, and this database was searched for patients with a diagnosis of subdural empyema who underwent surgery over an 11-year period between 1 January 2008 and 31 December 2018.

\section{Variables and Data Sources}

A retrospective case note review was performed on each patient to extract data pertaining to their demographics, presenting features, treatment, and clinical outcome at the time of discharge. Demographic data included age and gender. Presenting features included symptoms and signs of presentation, initial biochemical values (white cell count [WCC] and C-reactive protein [CRP]), and causative organism. Radiological data were gathered by a review of the presenting MRI and/or CT scans, assessing for whether the subdural collections were unilateral/bilateral/parafalcine only and additionally whether there was any parafalcine collection. Treatment data included surgical treatment including multiple procedures and use of cranioplasty, empirical antibiotic regime, and use of anti-seizure medication. Outcome data included modified Rankin Scale (mRS) at discharge, mortality, morbidity (neurological sequelae and persistent residual neurological deficits), and length of hospital stay.

\section{Microbiological Diagnoses}

A key determinant of the success of antibiotic therapy is acquiring a microbiological diagnosis. Our protocol involves initial culture and subculture of the intraoperative pus specimens from overnight enrichment in brain heart infusion and extended culture following prolonged enrichment in an anaerobic selective medium (Robertson's Cooked Meat broth). When initial samples are negative, they are processed using broad-range $16 \mathrm{~S}$ rRNA polymerase chain reaction (PCR) followed by specific PCRs for organisms including Streptococcus pneumoniae, Neisseria meningitidis, Streptococcus agalactiae, Streptococcus pyogenes, Enterobacterales group, and Staphylococcus aureus.

\section{Study Size and Statistical Methods}

Study size was based on, and limited to, the number of patients presenting to our department over an 11-year period. No specific power calculation was performed. Loss to follow-up was considered and it was decided that time of follow-up would be limited to length of inpatient hospital stay. Statistical analyses were performed on Microsoft Excel and MATLAB v2018b.

\section{Results}

\section{Demographics}

A total of 36 patients were treated over the 11-year inclusion period. Median age was 11.0 (range 0.2-15.8), and there was a bimodal age distribution with peaks at age $0-2$ and $10-12 ; 47.2 \%(17 / 36)$ were female (Fig. 1a). The most common presenting symptoms were fever $(55.6 \%)$ and headache (52.8\%) (Fig. 1b). Two-thirds $(66.6 \%, 24 / 36)$ had a CRP $>150$ and $22.2 \%(8 / 36)$ had a WCC $>20$. All patients underwent axial imaging prior to surgical treatment 91.6\% (33/36) underwent CT scan and 30.6\% (11/36) had an MRI scan. These showed subdural collections that were unilateral convexity in $72.2 \%(26 / 36)$, bilateral (bifrontal) in $16.7 \%(6 / 36)$, and parafalcine in $11.1 \%(4 / 36)$.

\section{Surgical Intervention}

In total, the 36 patients underwent 44 surgical procedures ( 5 patients had 2 procedures and 2 had 3 ), and the technique was at the discretion of the operating surgeon (classified into burr hole aspiration, craniotomy, or cra- 


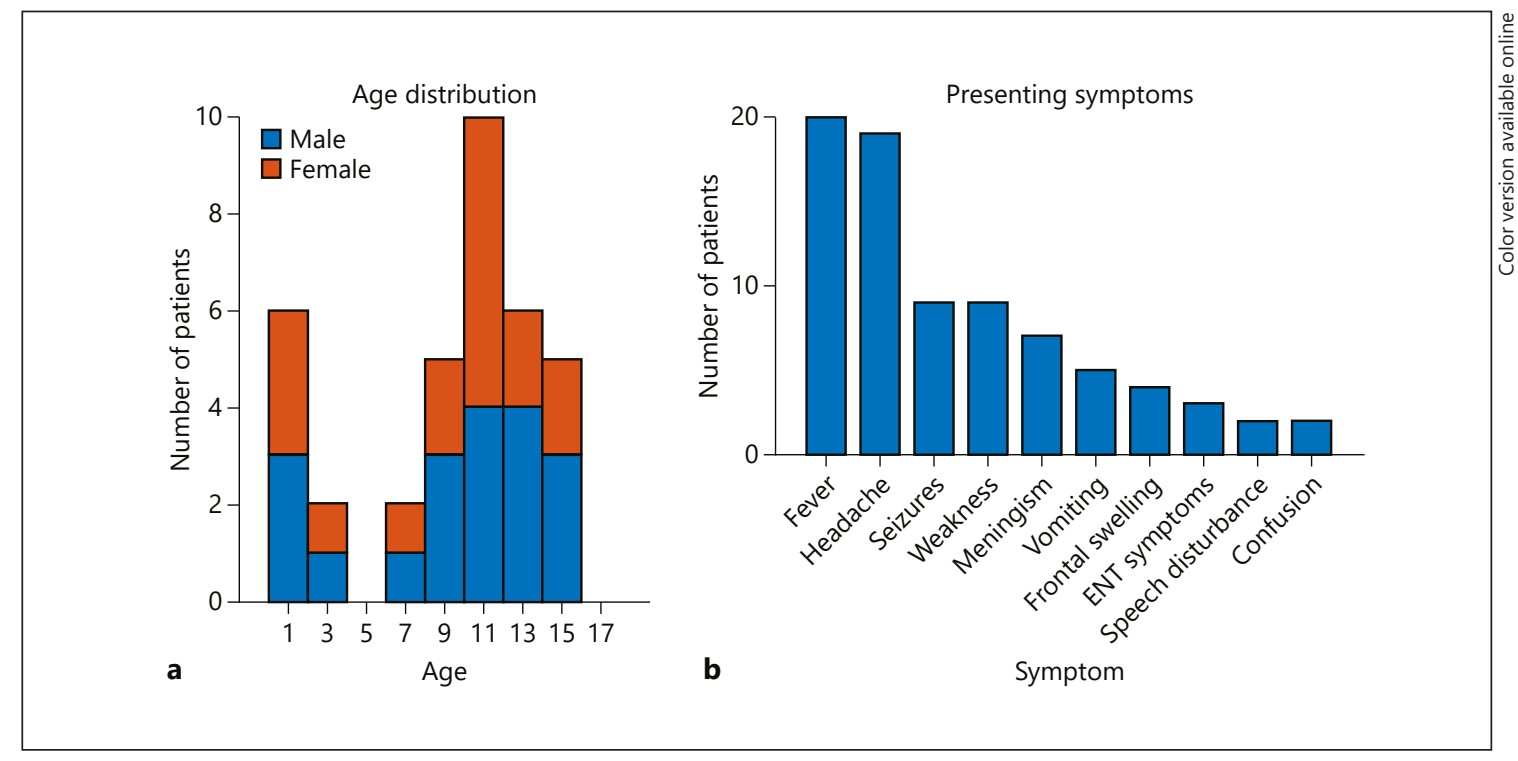

Fig. 1. a Age and sex distributions of the 36 patients, showing a bimodal distribution. b Presenting symptoms of the patients, with headache and fever being the most common.

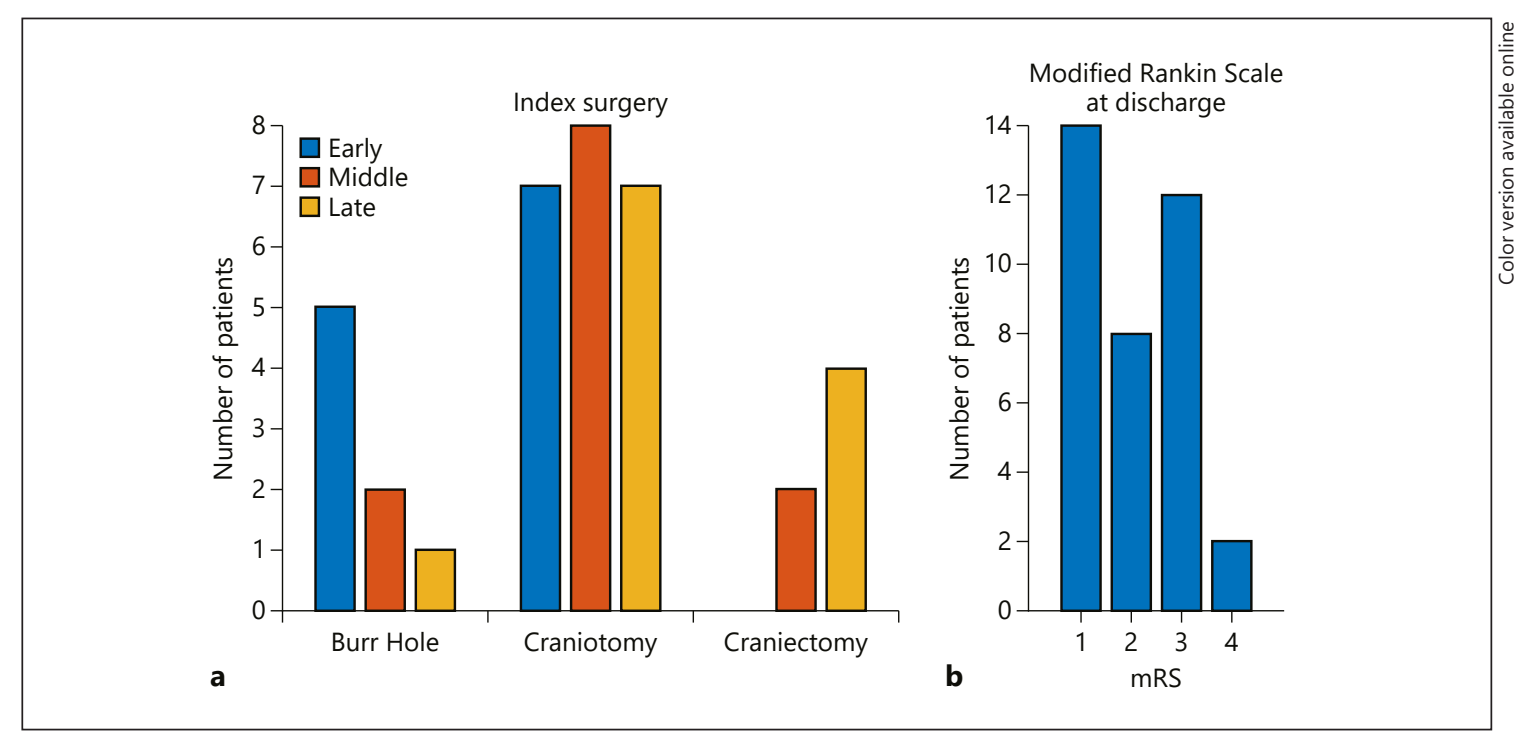

Fig. 2. a Surgical approach over time, illustrating a decreasing use of burr hole aspiration and increasing use of craniectomy over time. b Outcomes, as assessed by the mRS at discharge from the neurosurgical unit. mRS, modified Rankin Scale.

niectomy). In order to assess trends over time, the cohort was split into 3 based on case order (early, middle, and late). Over time, there was decreasing use of burr hole aspiration and increasing use of craniotomy as the index procedure (Fig. 2a). Four patients (11.1\%) underwent a concurrent ENT procedure (e.g., endoscopic sinus washout or mastoidectomy) as part of the index procedure.

\section{Microbiological Diagnosis}

All 36 patients achieved a microbiological diagnosis using our clinical protocol (see Methods for details). Twelve (33.3\%) were achieved from the initial or extended culture, 13 (36\%) from PCR, and $10(27.8 \%)$ that confirmed the same organisms on both culture and PCR; the final case was diagnosed by pneumococcal urinary antigen testing. The 
Table 1. Comparison of demographic and operative variables between the patients undergoing repeat surgery and those not undergoing repeat surgery in the cohort

\begin{tabular}{|c|c|c|c|c|}
\hline & $\begin{array}{l}\text { No repeat surgery } \\
(n=29)\end{array}$ & $\begin{array}{l}\text { Repeat surgery } \\
(n=7)\end{array}$ & $\begin{array}{l}\text { Total } \\
(n=36)\end{array}$ & $p$ value \\
\hline \multicolumn{5}{|l|}{ Age } \\
\hline Median (range) & $11.7(2.7-15.8)$ & $10.6(0.2-15.6)$ & & 0.39 \\
\hline \multicolumn{5}{|l|}{ Group } \\
\hline Early & 10 & 2 & 12 & \multirow{3}{*}{0.28} \\
\hline Middle & 11 & 1 & 12 & \\
\hline Late & 8 & 4 & 12 & \\
\hline \multicolumn{5}{|l|}{ Location of collection } \\
\hline Bilateral & 9 & 1 & 10 & \multirow{3}{*}{0.24} \\
\hline Parafalcine only & 5 & 0 & 5 & \\
\hline Unilateral & 15 & 6 & 21 & \\
\hline \multicolumn{5}{|l|}{ Any parafalcine collection? } \\
\hline Yes & 14 & 2 & 16 & \multirow{2}{*}{0.35} \\
\hline No & 15 & 5 & 20 & \\
\hline \multicolumn{5}{|l|}{ Presenting WCC } \\
\hline$<20$ & 22 & 6 & 28 & \multirow{2}{*}{0.57} \\
\hline$>20$ & 7 & 1 & 8 & \\
\hline \multicolumn{5}{|l|}{ Presenting CRP } \\
\hline$<150$ & 9 & 0 & 9 & \multirow[b]{2}{*}{0.08} \\
\hline$>150$ & 17 & 7 & 24 & \\
\hline \multicolumn{5}{|l|}{ Index operation type } \\
\hline Burr hole & 7 & 1 & 8 & \multirow{3}{*}{0.60} \\
\hline Craniotomy & 18 & 4 & 22 & \\
\hline Craniectomy & 4 & 2 & 6 & \\
\hline \multicolumn{5}{|l|}{ Concurrent ENT procedure } \\
\hline Yes & 4 & 0 & 4 & \multirow[b]{2}{*}{0.29} \\
\hline No & 25 & 7 & 32 & \\
\hline \multicolumn{5}{|l|}{ Microorganism } \\
\hline S. anginosus group & 15 & 3 & 18 & \multirow{3}{*}{0.31} \\
\hline S. anginosus group + other & 5 & 3 & 8 & \\
\hline Other & 9 & 1 & 10 & \\
\hline
\end{tabular}

Age was compared using a Kruskal-Wallis test and all other categorical variables using $\mathrm{X}^{2}$ tests. WCC, white cell count; CRP, C-reactive protein. most common pathogens were the Streptococcus anginosus group (S. anginosus, S. constellatus, and S. intermedius) $(72.2 \%, 26 / 36)$, of whom $8(22.2 \%)$ also had other concurrent organisms. Four (11.1\%) had other streptococcal species, including 1 case of Streptococcus pyogenes following spread from periorbital cellulitis. Six (16.7\%) had other organisms including 1 case of methicillin-resistant Staphylococcus aureus in a case of subdural empyema with associated brain abscess in a child returning from travel overseas.

\section{Risk Factors for Repeat Intervention}

Of the 7 patients who underwent repeat surgery (19.4\% of the overall cohort), 2 were in the early group, 1 in the middle, and 4 in the late group; one had burr hole aspiration as a primary procedure, 4 underwent craniotomy, and 2 underwent craniectomy (Table 1). Importantly, repeat surgery was for recollection of the subdural empyema (6 cases); in 1 patient, repeat surgery had to be performed to remove a bone flap from a craniotomy that subsequently became infected. In order to predict factors that may influence the need for repeat surgery, a range of clinical variables were chosen to assess whether there was difference in characteristics between those that did and did not require repeat surgery (Table 1). None of these factors were predictive, although a number of interesting observations were made. Firstly, repeat surgery was more likely in those with a presenting high $\mathrm{CRP}(>150, p=0.08)$, a potentially useful and readily available clinical biomarker. None of the neonates required repeat drainage and only 1 was $<11$ years old. None of the children that underwent a concurrent ENT washout required repeat surgery and that the majority of those that did require repeat surgery had S. anginosus 
species. As none of the factors were predictive in this univariate analysis, no further analysis was undertaken.

\section{Post-Operative Management}

Based on the culture and sensitivities, antibiotics were rationalised and continued for a median of 6 weeks (range $2-12)$ following surgery. Twenty-five $(69.4 \%)$ were started on antiepileptic medication for a combination of preoperative ( 9 patients) or post-operative (16 patients) seizures; routine prophylaxis of antiepileptic medication was not used. Phenytoin was used in 23 patients and levetiracetam in 2 ( 1 of whom was previously taking levetiracetam for complex partial seizures and the other due to a possible interaction between phenytoin and metronidazole). One patient had an adverse reaction to phenytoin (Stevens-Johnson syndrome), which was replaced with sodium valproate.

Additional management was based on the post-operative course. Five patients (13.8\%) developed venous sinus thrombosis and were anticoagulated for a median of 3 (range 1-5) months. Two (5.5\%) developed hydrocephalus, 1 requiring temporary and the other requiring permanent CSF diversion.

Median length of stay in the neurosurgical centre was 12 days (range 3-74), and there were no inpatient or procedure-related mortalities. The mRS was used to assess outcome at discharge, with $94.4 \%(34 / 36)$ being mRS 0-3 (Fig. 2b). Of note, both patients who were mRS 4 at discharge improved following discharge; one had a haemiparesis that almost completely resolved 1 year following discharge whilst the others' developmental outcome improved with no associated motor deficits 3 years following discharge. Neither had repeat surgery.

Six patients underwent cranioplasty of which 5 were attempted autologous and 1 titanium. There were $2 \mathrm{com}$ plications, both in the autologous group. One operation was abandoned as the bone flap, stored in the abdomen, was found to be infected, and therefore, a polyetheretherketone (PEEK) cranioplasty was inserted a few months later without complication. The other presented with a bone flap infection soon after autologous cranioplasty; this was also replaced by PEEK 6 months later but had to be removed due to recurrent infection.

\section{Discussion}

Our contemporary series of management of subdural empyema highlights some important changes in the presentation and management of this disease over time.
Firstly, in terms of demographics, the bimodal age distribution is in keeping with the previous observations, with peaks in infancy (secondary to meningitis) and older children (secondary to sinusitis or otitis media) [2-5]. Indeed, others have noted an increased incidence of both sinusitis and its intracranial complications over time, with the cause for this being unclear [6]. In our series, there were no iatrogenic subdural empyemas (secondary to prior neurosurgical intervention). Presenting CRP and WCC can be useful markers of the severity of infection; indeed, a high presenting CRP of $>150$ may be indicative of a more aggressive infection that may require repeat surgery $(p=0.08)$.

The range of causative organisms, with the majority being Streptococcus anginosus group (S. anginosus, S. constellatus, and S. intermedius), is again in keeping with previous series [2, 3, 6-8]. Importantly, all 36 patients had an organism isolated from the intraoperative samples, in contrast to Gupta et al. [3] who had a 12.9\% rate of "cryptogenic" infection in their mixed series of subdural and extradural infections. This is an important consideration in the overall outcome of these children as, despite surgery being an important part of the treatment, definitive management is through long-term antibiotic therapy and this can only be effectively guided if a microorganism is isolated. In our series, this was facilitated by robust culture and PCR processes that ensured accurate microbiological diagnoses in all patients, irrespective of whether antibiotic therapy was initiated prior to surgical intervention. Given these findings, our series provides evidence that antibiotic therapy should be commenced at diagnosis as there is no benefit of "increasing yield" in waiting for surgical intervention; although we have not been able to specify what proportion received antibiotics prior to surgery in this retrospective series, our routine management throughout the time-period was to commence antibiotics without delay. In addition, the spectrum of microbiological diagnoses provides evidence of which empirical antibiotics to commence at presentation and, given the results, we would advocate for a third-generation cephalosporin and metronidazole as first line, in agreement with other reports from similar cohorts [9].

Although the analysis to identify factors that may predict need for repeat surgery revealed no significant factors, some observations are worth highlighting. Although age was not different between the groups, none of the neonates required repeat surgery and only 1 patient requiring repeat surgery was $<11$ years old. In addition, none of those that underwent a concurrent ENT procedure (endoscopic sinus washout or mastoidectomy) un- 
derwent repeat surgery. This underscores the importance of source control; controlling the intracranial infection may be a life-saving procedure, but, in order to prevention re-exacerbation, we advocate close liaison with our ENT colleagues and an aggressive approach to source control.

The surgical approach and changes with time are also interesting to consider. Over time, at our institution, there has been a trend to be more invasive with the procedure, with reducing use of burr hole drainage and increasing use of craniectomy; craniotomy numbers have been stable (Fig. 2a). Whilst there is evidence that craniotomy is safe in these patients from the perspective of the bone flap not becoming infected [10], there were 2 cases of bone flap infection following craniectomy in our series. In addition, whilst both of these observations were not statistically significant, the rate of repeat surgery increased over time and increased with the more invasive procedures (Table 1). This may be an effect of the severity of the disease necessitating larger approaches. Similar observations have been made by Konar et al. [4], who found a non-significant increase in odds of poor outcome for craniotomy compared to burr hole drainage and this may indeed be a selection bias with the more severe cases requiring a craniectomy (due to intraoperative swelling precluding bone flap replacement) and also being at risk for requiring further interventions.

In terms of the outcomes, this series illustrates that, with prompt management, mortality can be avoided and favourable outcomes can be achieved with low levels of disability. However, specific complications need to be actively watched for. Although many have associated venous sinus thrombosis, the 5 patients $(13.8 \%)$ in our series completed uncomplicated courses of anticoagulant treatment with no other sequelae. CT or MR venography is a useful adjunct in these cases, although perhaps should be considered after surgical evacuation as there could be false positives from compression of the sinuses (as was the case in 1 of our patients). Our rate of seizures (69.4\%) was also a lot higher than previous series $[2,3,8]$. The question of whether or not this warrants more widespread prophylactic treatment with antiepileptic drugs rests on whether or not these early seizures predispose to longterm epilepsy, which we are unable to ascertain given the current data.

Cranioplasty surgery following craniectomy was performed using autologous, PEEK, and titanium implants. We observed 2 complications in this subgroup suggesting that autologous cranioplasty must be ap- proached with care but, given the limited number of patients in this subgroup, it is difficult to draw strong conclusions. Widdel et al. [11] previously described a technique to successfully treat an infected bone flap through vigorous surgical debridement. At our institution, we also ensure meticulous debridement of the bone flap; this is likely to be important in ensuring successful outcomes with the use of autologous bone flaps in subdural empyema.

\section{Limitations}

This is a retrospective observational study with limited patient numbers regarding a rare pathology in the paediatric population. Accordingly, our results should be interpreted with caution. Given the small number of patients in our study, it was not possible to find statistically significant risk factors that would reliably predict the need for re-operation. The choice for craniotomy/craniectomy or burr hole drainage was determined by the treating surgeon. This caused a selection bias. We were also only able to assess functional outcome at discharge as our service is a regional one and some children would get longer follow-up at their local hospitals; future studies would benefit from robust assessment of long-term functional and developmental outcomes. Randomised trials are not ethical or appropriate, and despite the inherent limitations, we feel our study provides a useful contribution to the limited current literature to inform the treating paediatric neurosurgeon.

\section{Conclusions}

Our case series shows that prompt surgical intervention followed by organism-specific antibiotic therapy results in favourable clinical outcomes in children presenting with subdural empyema. The importance of a comprehensive protocol including extended culture and PCR in achieving a microbiological diagnosis is stressed and, given that we achieved a diagnosis in all 36 cases, makes the case for prompt commencement of antibiotic therapy at diagnosis in these children. Repeat surgery was commoner in the older children. The trend towards larger index surgery with time was also associated with a higher re-operation rate. Concurrent ENT surgery may be an important factor in providing aggressive source control thereby reducing the need for re-operation. 


\section{Acknowledgements}

We would like to acknowledge the consultant neurosurgical team at GOSH (Owase Jeelani, Dominic Thompson, Martin Tisdall, Greg James, Kristian Aquilina, and Dulanka Silva) for their care of the patients included in this series.

\section{Statement of Ethics}

This study was registered as a service evaluation with the Great Ormond Street Hospital Clinical Audit Department. In line with the UK Health Research Authority guidelines, such studies are exempt from formal Regional Ethics Committee (REC) approval and individual patient consent is not required. This study was conducted ethically and in accordance with the World Medical Association Declaration of Helsinki.

\section{Conflict of Interest Statement}

The authors have no conflicts of interest to report.

\section{Funding Sources}

A.C. is supported by a Great Ormond Street Hospital (GOSH) Children's Charity Surgeon Scientist Fellowship. This work has been supported by the GOSH-National Institute of Health Research Biomedical Research Centre.

\section{Author Contributions}

M.Z.T. and F.T.R. conceived the idea. F.T.R., M.O.I., G.S., and A.C. collected and analysed the data. All authors were involved in the drafting and review of the manuscript and approved the final manuscript prior to submission.

\section{Data Availability Statement}

Anonymized data can be made available from the corresponding author on reasonable request.

\section{References}

1 Mauser HW, Van Houwelingen HC, Tulleken CA. Factors affecting the outcome in subdural empyema. J Neurol Neurosurg Psychiatry. 1987 Sep;50(9):1136-41.

2 Muzumdar D, Biyani N, Deopujari C. Subdural empyema in children. Childs Nerv Syst. 2018 Oct;34(10):1881-7.

3 Gupta S, Vachhrajani S, Kulkarni AV, Taylor MD, Dirks P, Drake JM, et al. Neurosurgical management of extraaxial central nervous system infections in children: clinical article. J Neurosurg Pediatr. 2011 May;7(5):441-51.

4 Konar S, Gohil D, Shukla D, Sadashiva N, Uppar A, Bhat DI, et al. Predictors of outcome of subdural empyema in children. Neurosurg Focus. 2019;47(2):E17.
5 Cole TS, Clark ME, Jenkins AJ, Clark JE. Pediatric focal intracranial suppuration: a UK single-center experience. Childs Nerv Syst. 2012 Dec;28(12):2109-14.

6 McNeil JC, Dunn JJ, Kaplan SL, Vallejo JG. Complications of otitis media and sinusitis caused by Streptococcus anginosus group organisms in children. Pediatr Infect Dis J. 2020 Feb;39(2):108-13.

7 Makwana M, Merola JP, Bhatti I, Patel CK, Leach PA. Towards improved outcome in children treated surgically for spontaneous intracranial suppuration in South Wales. Br J Neurosurg. 2021 Jan:1-4. Epub ahead of print.
8 Otto WR, Paden WZ, Connors M, Joerger T, Buzi A, Rizzi M, et al. Suppurative intracranial complications of pediatric sinusitis: a single-center experience. J Pediatric Infect Dis Soc. 2021 Apr;10(3):309-16.

9 van der Velden FJS, Battersby A, Pareja-Cebrian L, Ross N, Ball SL, Emonts M. Paediatric focal intracranial suppurative infection: a UK single-centre retrospective cohort study. BMC Pediatr. 2019 Apr;19(1):130.

10 Dandurand C, Schaurich C, Tamber M, McDonald P, Steinbok P. Immediate replacement of bone flap after craniotomy for empyema in children. Childs Nerv Syst. 2021;37(2):475-9.

11 Widdel L, Winston KR. Pus and free bone flaps: clinical article. J Neurosurg Pediatr. 2009 Oct;4(4):378-82. 